\title{
ANTIMICROBIAL SUSCEPTIBILITY AND DETECTION METHODS FOR THE EXTENDED-SPECTRUM $\beta$-LACTAMASES PRODUCING ENTEROBACTERIACEAE FROM CLINICAL SAMPLES
}

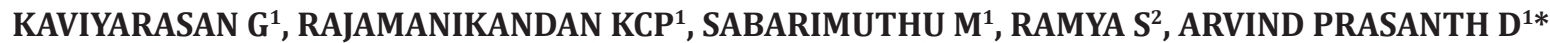 \\ ${ }^{1}$ Department of Microbiology, Medical Microbiology Laboratory, Periyar University, Salem, Tamil Nadu, India. ${ }^{2}$ Department of \\ Microbiology, Shri Sakthikailassh Women's College, Tamil Nadu, India. Email: prasanthviji@gmail.com
}

Received: 19 December 2017, Revised and Accepted: 22 January 2018

\begin{abstract}
Objectives: Detection of extended-spectrum $\beta$-lactamases (ESBLs) is crucial for the infection control and antibiotic choice in healthcare settings. The aim of this study is to develop a standardized, inexpensive, and simple approach that is able to detect ESBL-producing Enterobacteriaceae isolates.

Methods: Isolates those were resistant to at least one of the three indicator cephalosporins (cefotaxime, cefpodoxime, and ceftazidime) were tested for ESBL production using the double disc synergy test (DDST), combined disc synergy test (CDST) test and genotypic detection of the responsible gene for the ESBL.
\end{abstract}

Result: From 64 isolates, 28 were resistant to cephalosporins. In 28 isolates, 23 were positive in CDST but in the DDST 18 were showing ESBL positive. 10 were positive in both CDST and DDST.

Conclusion: Resistance to cephalosporins, which are the drug choice to treat mixed bacterial infections by the Enterobacteriaceae of which disseminate rapidly being plasmid mediated. Hence, it is necessary that rapid detection of ESBL should be done and immediate infection control measures should be implemented to prevent their dissemination.

Keywords: Cephalosporins, Enterobacteriaceae, Extended-spectrum $\beta$-lactamases, TEM, SHV.

(c) 2018 The Authors. Published by Innovare Academic Sciences Pvt Ltd. This is an open access article under the CC BY license (http://creativecommons. org/licenses/by/4. 0/) DOI: http://dx.doi.org/10.22159/ajpcr.2018.v11i5.24356

\section{INTRODUCTION}

Beta-lactam group of antibiotics is one of the most common antibacterial agents used to treat the mixed bacterial infections in both developed and developing countries including India [1]. The production of $\beta$-lactamases such as extended-spectrum $\beta$-lactamases (ESBLs), AmpC, and carbapenemase is the most common cause of bacterial resistance against beta-lactam antibiotics. In between the $\beta$-lactamase enzymes, ESBLs are rapidly growing group of $\beta$-lactamases that mediate resistance to extended-spectrum cephalosporins and monobactams but not affected by cephamycins (cefoxitin, cefotetan, and cefmetazole) and carbapenems (imipenem, meropenem, ertapenem, doripenem, etc.) [1,2].

ESBLs are showing resistance to penicillin and narrow-spectrum cephalosporin by hydrolyzing the beta-lactam ring and are inhibited by $\beta$-lactamase inhibitors such as clavulanic acid, sulbactam, and tazobactam [4-6]. These are commonly produced by members of the Enterobacteriaceae, namely, Escherichia coli, Klebsiella pneumoniae, and Proteus [7,8] other bacterial species in the families of Enterobacteriaceae and Pseudomonadaceae are also known to produce these enzymes [9]. These bacteria are a serious problem for infection control by practitioners. Hence, early detection and identification of these resistance enzymes will enable optimum antimicrobial therapy, and ensure the timely introduction of infection control procedures to prevent further spread as these infections $[10,11]$.

ESBLs are often associated with plasmids harboring resistance genes to other antimicrobial classes, resulting in multidrug-resistant isolates thereby facilitating their transfer to different hosts $[12,13]$. The first ESBLs have evolved from native $\beta$-lactamases TEM and SHV by a genetic mutation. However, from the 2000s, a novel type of ESBL called CTX-M has emerged worldwide [14-16].
Control of infection by these microorganisms is a major challenge that requires accurate and fast ESBL detection in clinical microbiology laboratories. Hence, the rapid characterization of these isolates is very important in the treatment of hospitalized patients. The aim of this present study is to develop a standardized, inexpensive and simple approach that is able to detect ESBL-producing Enterobacteriaceae isolates in a routine clinical laboratory.

\section{METHODS}

This conceivable study was conducted at the Medical Microbiology Laboratory of Periyar University, Salem, Tamil Nadu, India. A total of 78 clinical samples was collected from the hospitals surrounding Salem. The clinical samples urine, swabs, sputum, blood, and body fluid were collected from patients presenting at the local hospitals between the periods of January 2017 and February 2017. The samples were inoculated onto blood agar (BA) and MacConkey agar plates and inoculated plates were incubated at $37^{\circ} \mathrm{C}$ for $24 \mathrm{~h}$. The isolates presenting at a significant number (colony counts $\geq 105 \mathrm{CFU} / \mathrm{ml}$ ) were identified according to standard procedures.

\section{Antimicrobial susceptibility tests}

Antibiotic susceptibility of the clinical isolates was tested by the Kirby-Bauer disc diffusion method on Mueller Hinton Agar following CLSI guidelines using antibiotic discs containing penicillin (10 mcg), amoxicillin $(30 \mathrm{mcg})$, ampicillin $(10 \mathrm{mcg})$, ceftriaxone $(30 \mathrm{mcg})$, ceftazidime $(30 \mathrm{mcg})$, cefotaxime $(30 \mathrm{mcg})$, gentamycin $(10 \mathrm{mcg})$, tetracycline (30 $\mathrm{mcg})$, ciprofloxacin $(5 \mathrm{mcg})$, and meropenem (10 mcg).

\section{$\beta$-lactamases assay}

This assay was done by plate and tube method to detect. The production of $\beta$-lactamases enzymes by the cephalosporins resistant isolates was tested by plate and tube method. 


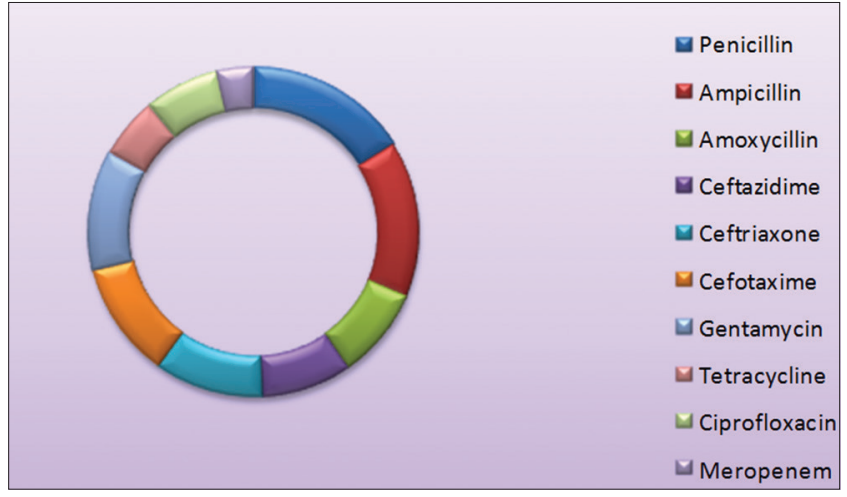

Fig. 1: Resistant profile of pathogens

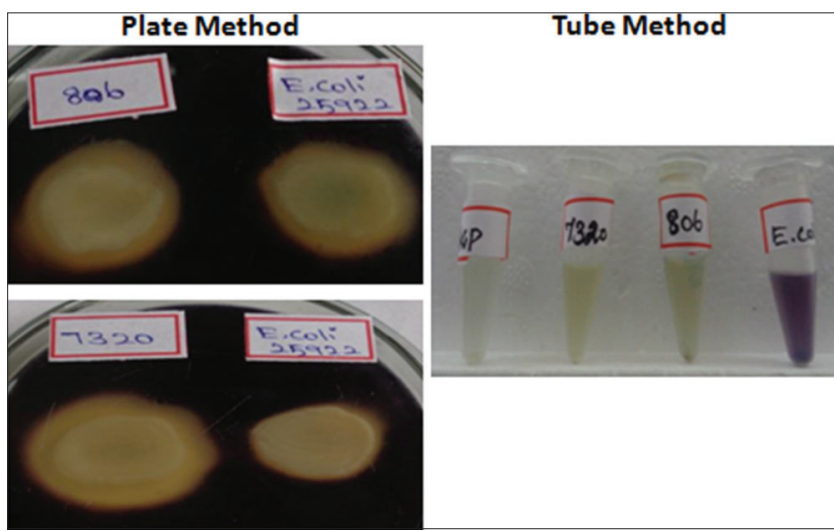

Fig. 2: Detection of $\beta$-lactamase

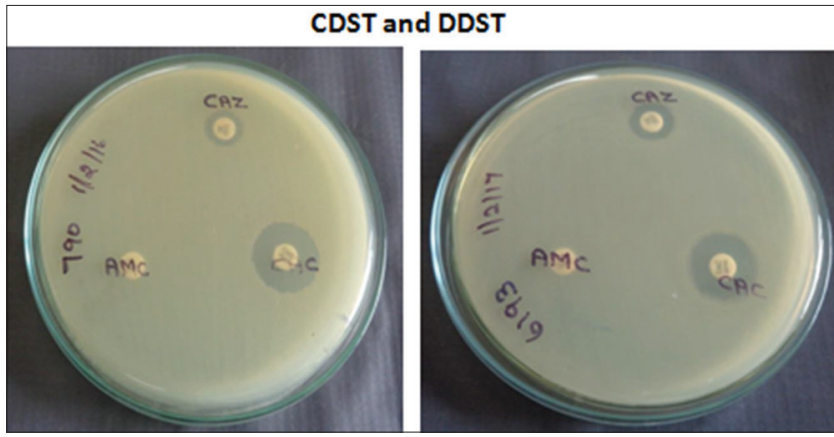

Fig. 3: Phenotypic detection of extended-spectrum $\beta$-lactamases

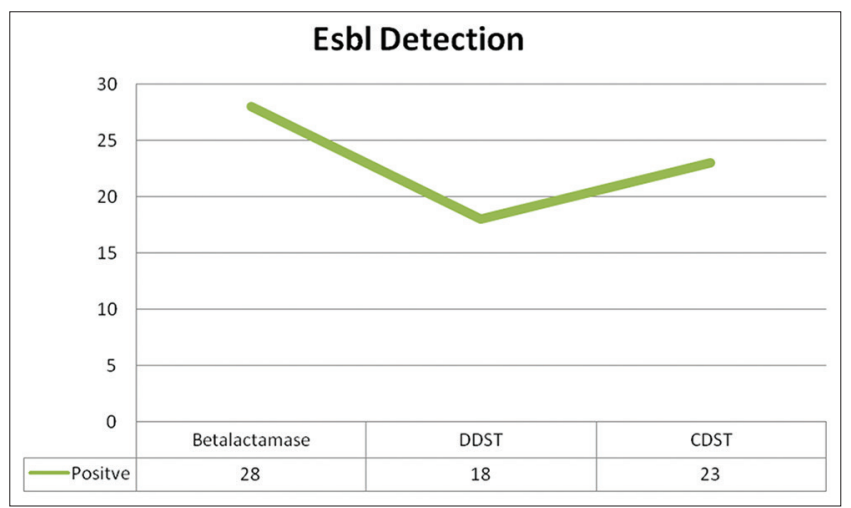

Chart 1: Detection of $\beta$-lactamase and extended-spectrum $\beta$-lactamases

\section{Plate method}

The $\beta$-lactamase assay by plate method was done following the method of Kumar et al., 2013. A loopful culture of the test organism was spot inoculated on Mueller Hinton agar (MHA) containing 1\% starch and penicillin $(10,000$ units). After incubation of plates at $37^{\circ} \mathrm{C}$ for $18-24 \mathrm{hrs}$, the plates were flooded with freshly prepared phosphate buffered saline (PBS) containing potassium iodide, iodine. The appearance of the clear, colorless zone around the bacterial growth is an indication of $\beta$-lactamase production. The $\beta$-lactamase converts penicillin to penicillin acid, which reduces iodine to iodide monitored through decolorization of the starch-iodine complex [17].

\section{Tube method}

The $\beta$-lactamase assay by tube method was done following the method of Alaa et al. The starch indicator was prepared by dissolving $1 \mathrm{~g}$ of starch powder in $100 \mathrm{ml}$ of boiling water. The iodine reagent was prepared by dissolving $2 \mathrm{~g}$ of $\mathrm{KI}$ (potassium iodide), $1 \mathrm{~g}$ of iodine in $100 \mathrm{ml}$ of distilled water (D.W). The solution was then stirred vigorously for $15 \mathrm{~min}$ and stored in brown bottles. A solution of 10000 units of benzylpenicillin per $\mathrm{ml}$ was prepared in phosphate buffer $\mathrm{pH} 70$. This was made by dissolving $0.6 \mathrm{~g}$ of penicillin G powder in $60 \mathrm{ml}$ of PBS. The volume was made up to $100 \mathrm{ml}$ with D.W and then sterilized by Millipore $(0.22 \mu \mathrm{m})$ filters. The test was conducted by adding a loop full of overnight grown culture (of the tested bacteria) to $0.5-1 \mathrm{ml}$ of the penicillin $\mathrm{G}$ solution. The tube was left at $37^{\circ} \mathrm{C}$ for $30 \mathrm{~min}$. Thereafter, 2 drops of the starch solution were added, followed by 1 drop of iodine reagent. A color change from dark blue to colorless was noted over the next $10 \mathrm{~min}$. The change of the black color to colorless solution indicated positive test [18].

\section{ESBL detection}

The clinical isolates showing reduced susceptibility to ceftazidime (zone diameter of $<22 \mathrm{~mm}$ ), ceftriaxone (zone diameter of $<25 \mathrm{~mm}$ ), or cefotaxime (zone diameter of $<27 \mathrm{~mm}$ ) as recommended by CLSI guidelines (2010), were screened for confirmation of ESBL production by following two different methods, namely, double disc synergy test (DDST) and combined disc synergy test (CDST).

\section{DDST}

Amoxicillin/clavulanic acid $(20 \mu \mathrm{g} / 10 \mu \mathrm{g})$ and third generation cephalosporin discs were placed at a distance of $20 \mathrm{~mm}$ from center to center of the lawn cultures on Mueller Hinton agar plates. The plates were incubated at $37^{\circ} \mathrm{C}$ overnight. An enhancement in the zone of inhibition of cephalosporins toward the amoxicillin/clavulanic acid disc was considered a positive result for ESBL $[19,20]$.

\section{CDST}

The phenotypic confirmation of ESBL producers was carried out by as per CLSI (2010) guidelines. A disc of ceftazidime $(30 \mu \mathrm{g})$ alone and a disc of ceftazidime + clavulanic acid $(30 \mu \mathrm{g} / 10 \mu \mathrm{g})$ were used in this study. Both the discs were placed at least $25 \mathrm{~mm}$ apart, center to center, on a lean culture of the test isolate on to MHA plate and incubated overnight at $37^{\circ} \mathrm{C}$. Difference in zone diameters with and without clavulanic acid was measured. $\mathrm{A} \geq 5 \mathrm{~mm}$ increase in the zone diameter of inhibition for any of the antimicrobial agents tested in combination with clavulanic acid compared to the zone diameter of inhibition when tested alone was considered as confirmatory for ESBL production [21-23].

To check the quality of these tests the ESBL negative strain of $E$. coli ATCC 25922 and ESBL positive strain K. pneumoniae ATCC 700603 were used as a standard control for both methods.

\section{Genotypic detection of ESBL}

The genotypic identification of ESBL producers was carried out by isolation of plasmid DNA. Isolation of plasmid DNA was carried out by alkaline lysis procedure (Sadasivam and Manickam, 2008) with some modification. $1 \mathrm{ml}$ of an overnight culture was transferred into an eppendorf tube. The cells were sedimented by centrifuging briefly 
(5000 RPM) in the microfuge and the supernatant was drained off. The pellet was resuspended by adding $100 \mu \mathrm{L}$ of solution A and the contents were mixed by a vortex. Then, $100 \mu \mathrm{L}$ solution B was added and mixed well by inverting the content ( $4-5$ times). To the above viscous content, $100 \mu \mathrm{L}$ of solution $\mathrm{C}$ was added, and the content was inverted 4-5 times to get mixed, the bulk of genomic DNA and other cell debris will precipitate into a viscous clump. It was centrifuged at 12000 RPM in a micro centrifuge, and the clamp was removed. The clear list (supernatant) was transferred to another eppendrof tube. $150 \mu \mathrm{L}$ of $100 \%$ isopropanol was added and mixed well and centrifuged the content at $12000 \mathrm{rpm}$ for $30 \mathrm{~min}$. The supernatant was drained off and $150 \mu \mathrm{L}$ of absolute alcohol was added and centrifuged the content at $10000 \mathrm{rpm}$ for $20 \mathrm{~min}$. The supernatant was drained off and dissolved the DNA pellets with $20 \mu \mathrm{L}$ TE buffer. The extracted Plasmid DNA was confirmed by running of agarose gel electrophoresis.

Isolates were screened for the resistance genes SHV, TEM, CTX-M, and OXA by a multiplex polymerase chain reaction (PCR) assay by following the protocol of Hong Fang et al., (2008) (Table 1). PCR amplification reactions were performed in a volume of $25 \mu \mathrm{L}$ containing $12.5 \mu \mathrm{L}$ of $2 \mathrm{x}$ Promega PCR Master Mix (USA), $0.2 \mu \mathrm{M}$ concentrations of each primer, and $2 \mu \mathrm{L}$ of DNA template. The cycling parameters were as follows: Initial denaturation at $95^{\circ} \mathrm{C}$ for $15 \mathrm{~min}$; followed by 30 cycles of $94^{\circ} \mathrm{C}$ for $30 \mathrm{~s}, 62^{\circ} \mathrm{C}$ for $90 \mathrm{~s}$, and $72^{\circ} \mathrm{C}$ for $60 \mathrm{~s}$; and with a final extension at $72^{\circ} \mathrm{C}$ for $10 \mathrm{~min}$. The amplified PCR products were subjected to electrophoresis at a $1.5 \%$ agarose gel in 1 XTBE buffer. A 100 bp ladder molecular weight marker (Helini, India) was used to measure the molecular weights of amplified products.

\section{RESULTS}

A total of 64 strains were isolated in significant numbers from collecting clinical samples. Antibiogram revealed that 54 (84\%) isolates were resistant to penicillin, $52(81 \%)$ isolates were resistant to ampicillin, and $30(46 \%)$ isolates were resistant to amoxycillin. The resistance to ceftazidime, ceftriaxone, cefotaxime, and gentamycin was

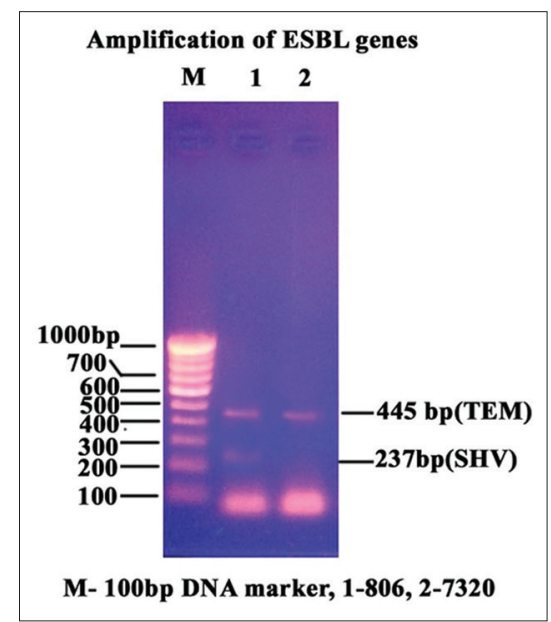

Fig. 4: Genotypic detection of extended-spectrum $\beta$-lactamases in between $40 \%$ and $60 \%$, resistance to tetracycline and ciprofloxacin was noted in between $30 \%$. Minimal resistance of $18 \%$ was noted to the drug meropenem, 28 isolates were selected for the detection of ESBL production and $\beta$-lactamase production, which as they showed resistance to ceftazidime, ceftriaxone, and cefotaxime (Fig. 1).

\section{ESBL detection}

The result of plate and tube methods showed that all the 28 isolates were $\beta$-lactamase producers as confirmed by both the methods. The CDST was found to be the best method for the detection of ESBL when using the DDST. Out of 28 isolates, 23 were positive in CDST but in the DDST 18 were showing ESBL positive (Figs. 2 and 3, Chart 1).

\section{Genotypic detection of ESBL isolates}

Of the 28 cephalosporin resistant clinical isolates, 10 isolates were ESBL positive by both CDST and DDST as they produced $\beta$-lactamase enzyme. 2 isolates were subjected to genotypic characterization by multiplexPCR for the presence of TEM, SHV, OXA, and CTXm gene. Strain 806 contains TEM and SHV then the strain 7320 contains TEM only (Fig. 4).

\section{DISCUSSION}

The infections caused by cephalosporin-resistant Gram-negative bacteria that produce an extended spectrum of $\beta$-lactamase enzymes have been reported with an increasing frequency in intensive care units and are associated with significant morbidity and mortality. Initially, this enzyme was produced by members of Enterobacteriaceae and some non-fermenters which hydrolyzed the most of $\beta$-lactam antibiotics.

In our study out of the 64 isolates, about 50 isolates showed resistant to penicillin and ampicillin, and about 30 isolates showed resistance to ceftazidime, ceftriaxone, and cefotaxime. Only 12 isolates showed resistant to meropenem. $\beta$-lactamase assay was done by plate and tube method all the 28 isolates produced $\beta$-lactamase enzyme in both methods. Kumar et al., 2013 found similar results, among 180 MDR Acinetobacter spp. 147 were positive for beta-lactamase assay method. Only $147 \beta$-lactamase positive isolates were subjected to confirm beta-lactamase mediated resistance mechanisms, and we found ESBL 84 (57.14\%), AmpC 105 (71.42\%), and MBL 43 (29.25\%) were confirmed by the phenotypic methods. Comparison of DDST and CDST phenotypic methods for detection of ESBL, the CDST was screened 23 ESBL positive isolates from 28 isolates which showed resistant to cephalosporins.

ESBL production was observed by Ranjini et al., 2015, in the $28.84 \%$ of the study subjects and $27.52 \%$ of the total isolates. The CDM detected $66.66 \%$ ESBLs among the ceftazidime-resistant strains compared to $55.55 \%$ by the DDST. ESBLs were more prevalent in Proteus mirabilis $(38.88 \%)$, Klebsiella spp. (30.76\%), and E. coli $(24 \%)$ while no ESBL production was seen in Citrobacter spp. and Agrawal et al. also delivered the same findings the ceftazidime, ceftriaxone, and cefotaxime resistant isolates were tested for the ESBL production in this study $84 \%$ of the isolates were ESBL positive in the phenotypic method. In Shah et al. revealed that both ertapenem and meropenem showed very low MICs against ESBL producing organisms. We found that piperacillin-tazobactam was also effective against ESBL producers, and its effectiveness is $96.29 \%$ for K. pneumoniae and $100 \%$ for E. coli.

Table 1: The forward and reverse primer sequence of SHV, TEM, CTXm, and OXA genes with amplification size

\begin{tabular}{lll}
\hline Gene name & Primer sequence & Amplified size (bp) \\
\hline SHV & CTT TAT CGG CCC TCA CTC AA & 237 \\
\multirow{2}{*}{ TEM } & AGG TGC TCA TCA TGG GAA AG & 445 \\
& CGC CGC ATA CAC TAT TCT CAG AAT GA & \\
CTXm & ACG CTC ACC GGC TCC AGA TTT AT & 593 \\
\multirow{2}{*}{ OXA } & ATG TGC AGY ACC AGT AAR GTK ATG GC & 813 \\
& TGG GTR AAR TAR GTS ACC AGA AYC AGC GG & \\
\hline
\end{tabular}


In our study, $82 \%$ of the ESBL producing organisms were sensitive to meropenem.

Multiplex PCR results showed the presence of TEM gene in both 806 and 7320 isolates. In case of 806 TEM and SHV were present. In a previous study by Karimian in Iran, the frequency of blaTEM and blaCTX-M genes in the 83 ESBL producing E. coli isolates was $27(32.5 \%)$ and $72(86.7 \%)$, respectively. However, 24 isolates (28.9\%) were indicated as having both genes. All E. coli strains harboring blaTEM were resistant to ceftazidime and cefotaxime, respectively, and ESBL production was observed in 59 (40.7\%) strains, comprising blaCTX- M-such as genes in $22(37.3 \%)$ and blaTEM in $42(71.2 \%)$ strains.

\section{CONCLUSION}

From the results of this study, it can be concluded that uncontrolled use of cephalosporin groups appears to be a potential risk factor for the emergence of ESBL producing strains of Enterobacteriaceae which poses a global challenge to microbiologist and clinicians. ESBLproducing bacteria have found their way out of the hospital, which could be considered as a risk factor in society. The monitoring and thoughtful usage of extended-spectrum cephalosporins, periodic observation of antibiotic resistance patterns will help to prevent the entry of ESBL producers into the environment. There is a need for the limitations and detected in the lab communication between microbiologist and physicians are the way control of the resistance.

\section{REFERENCES}

1. Zhao $\mathrm{WH}, \mathrm{Hu} \mathrm{ZQ}$. Beta-lactamases identified in clinical isolates of Pseudomonas aeruginosa. Crit Rev Microbiol 2010;36:245-58.

2. Garrec H, Drieux-Rouzet L, Gilmard J, Jarlier V, Robert J. Comparison of nine phenotypic methods for detection of extended spectrum b-lactamase production by Enterobacteriaceae. J Clin Microbiol 2011;49:1048-57.

3. Paterson DL, Bonomo RA. Extended-spectrum beta-lactamases: A clinical update. Clin Microbiol Rev 2005;18:657-86.

4. Mehrgan H, Rahbar M. Prevalence of extended-spectrum b-lactamase producing Escherichia coli in a tertiary care hospital in Tehran, Iran. Int J Antimicrob Agents 2008;31:147-51.

5. Pitout JD. Infections with extended-spectrum b-lactamase-producing Enterobacteriaceae: Changing epidemiology and drug treatment choices. Drugs 2010;70:313-33.

6. Rossolini GM, D'Andrea MM, Mugnaioli C. The spread of CTXM-type extended-spectrum beta-lactamases. Clin Microbiol Infect 2008;14 Suppl 1:33-41.

7. Canton R. Epidemiology and evolution of beta-lactamases. In: Baquero FN, Cassell G, Gutiérrez-Fuentes J, editors. Evolutionary Biology of Bacterial and Fungal Pathogens. Washington: ASM Press; 2008. p. 249-70.

8. Dziri R, Klibi N, Alonso CA. Characterization of extended-spectrum $\beta$-lactamase (ESBL)-producing Klebsiella, Enterobacter, and
Citrobacter obtained in environmental samples of a Tunisian hospital. Diagn Microbiol Infect Dis 2016;86:190-3.

9. Ranjini CY, Rangasamy VR. Detection of ESBL and plasmid-mediated AmpC beta lactamases among the Gram-negative bacteria isolates in diabetic foot ulcer infections. Community Acquir Infect 2015;2:57-61.

10. Kumar E, Usha K, Ramana BV, Chaudhury M, Sai Gopal DV. Prevalence of various B-lactamase (Esbl, Ampc and Mbl) producing multidrug resistant clinical isolates of Acinetobacter Spp. in a tertiary care hospital. Asian J Pharm Clin Res 2013;6:28-31.

11. Agrawal P, Ghosh AN, Kumar S. Prevalence of extended spectrum $\beta$-lactamases among E. Coli and K. Pneumoniae isolates in a tertiary care hospital. Indian J Pathol Microbiol 2008;51:139-42.

12. Kinal S, Summaya AM. Susceptibilities of Esbl-producing Enterobacteriaceae to ertapenem, meropenem and Piperacillintazobactam. Natl J Med Res 2012;2:223-5.

13. Karimian M, Rostamzad A, Shoaei P. Extended spectrum b-lactamase producing strains of Escherichia coli in hospitalized children in Isfahan, Iran. Avicenna J Clin Microbiol Infect 2015;2:270-96.

14. CLSI. Performance Standards for Antimicrobial Disk Susceptibility test; Approved Standard. CLSI Document M02-A11. $7^{\text {th }}$ ed. Wayne, PA: Clinical and Laboratory Standards Institute; 2010.

15. Sadasivam S, Manickam A. Biochemical Methods. $3^{\text {rd }}$ ed. New Delhi, India: New Age International Publishers; 2008.

16. Sahu MC, Dubey D, Rath S, Debata NK, Padhy RN. Multidrug resistance of Pseudomonas aeruginosa as known from surveillance of nosocomial and community infections in an Indian teaching hospital. J Pub Health 2012;20:413-23.

17. Jacoby GA, Munoz-Price LS. The new beta-lactamases. N Engl J Med 2005;352:380-91.

18. Bin C, Hui W, Renyuan Z, Yongzhong N, Xiuli X, Yingchun X, et al. Outcome of cephalosporin treatment of bacteremia due to CTX-M-type extended-spectrum beta-lactamase-producing Escherichia coli. Diagn Microbiol Infect Dis 2006;56:351-7.

19. Bhambri V. Significance of cse-1034 (elores ${ }^{\mathrm{TM}}$ ) in treatment of urinary tract infections due to multi-drug resistant extended spectrum beta-lactamases positive Escherichia coli. Asian J Pharm Clin Res 2016;9:12-3

20. Nivas D, Ramesh N, Krishnakumar V, Rajesh P, Solomon EK, Kannan VR. Distribution, isolation and characterization of lytic bacteriophages against Multi-drug resistant and extended-spectrum of $\beta$-lactamase producing pathogens from hospital effluents. Asian J Pharm Clin Res 2015;8:384-9.

21. Vinodhini R, Moorthy K, Palanivel P, Punitha T, Saranya S, Bhuvaneshwari M, Kanimozhi C. Detection and antimicrobial susceptibility pattern of esbl producing gram negative bacteria. Asian J Pharm Clin Res 2014;7:243-7.

22. Murugan N, Malathi J, Therese KL, Madhavan HN. Antimicrobial susceptibility and prevalence of Extended spectrum betalactamase (sbl) and Metallo betalactamase (Mbl) and its co-existence among Pseudomonas aeruginosa recovered from ocular infections. Int J Pharm Pharm Sci 2015;7:147-51

23. Saxena S, Banerjee G, Garga R, Singh M, Verma SK, Kushwaha RA. Esbl, Mbl And Amp C-B lactamases produced by superbugs: An emerging threat to clinical therapeutics. Int $\mathrm{J}$ Pharm Pharm Sci 2015;7:353-6 